\title{
Statistical theory of nonadiabatic transitions
}

\author{
A. A. Neufeld ${ }^{\text {a) }}$ \\ Abteilung für Spektroskopie und Photochemische Kinetik, Max-Planck-Institut für Biophysikalische Chemie, \\ Am Fassberg, D-37077 Göttingen, Germany
}

(Received 8 April 2004; accepted 9 February 2005; published online 28 April 2005)

\begin{abstract}
Based on results of the preceding paper, and assuming fast equilibration in phase space to the temperature of the surrounding media compared to the time scale of a reaction, we formulate a statistical theory of intramolecular nonadiabatic transitions. A classical mechanics description of phase space dynamics allows for an ab initio treatment of multidimensional reaction coordinates and easy combination with any standard molecular dynamics (MD) method. The presented approach has several features that distinguishes it from existing methodologies. First, the applicability limits of the approach are well defined. Second, the nonadiabatic transitions are treated dynamically, with full account of detailed balance, including zero-point energy, quantum coherence effects, arbitrarily long memory, and change of the free energy of the bath. Compared to popular trajectory surface hopping schemes, our MD-based algorithm is more efficient computationally, and does not use artificial $\mathrm{ad}$ hoc constructions like a "fewest switching" algorithm, and rescaling of velocities to conserve total energy. The enhanced capabilities of the new method are demonstrated considering a model of two coupled harmonic oscillators. We show that in the rate regime and at moderate friction the approach precisely reproduces the free-energy-gap law. It also predicts a general trend of the reaction dynamics in the low friction limit, and is valid beyond the rate regime. (C) 2005 American Institute of Physics. [DOI: 10.1063/1.1884516]
\end{abstract}

\section{INTRODUCTION}

The separation of electron and nuclear motions in molecules provided by an adiabatic Born-Oppenheimer (BO) approximation $^{1}$ plays a fundamental role in theoretical studies of chemical dynamics. The large mass difference between electrons and nuclei is reflected in different time scales of motion. In a zero-order approximation, nuclei in a molecule may be considered as "frozen," and diagonalization of the electronic Hamiltonian yields a set of corresponding eigenvalues, which depend parametrically on the (fixed) positions of nuclei, and are frequently called the adiabatic potential energy surface (PES). The next terms of the expansion with respect to the ratio of electron to nuclear mass couples the adiabatic PESs, and these terms are called nonadiabatic couplings.

Many reactions proceeding in the ground electronic state are accurately described within the adiabatic BO approximation. $^{2-4}$ It may, however, be violated when the energy splitting between adiabatic PESs are comparable to or smaller than the corresponding nonadiabatic couplings, and, therefore, the latter cannot be accounted perturbatively. Typical examples are reactive scattering at high energies, 5,6 and reactions involving electronically excited states. ${ }^{7-9}$ Fairly often, the breakdown of the $\mathrm{BO}$ approximation occurs in highly localized regions of a PES, which allows to account for the nonadiabatic effects introducing a transition probability between the adiabatic PESs, when the system crosses the region of nonadiabaticity. 10,11

There exist several basic models, ${ }^{12-19}$ which estimate the

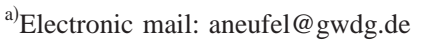

probability of the nonadiabatic transitions for many situations of interest (see also Ref. 20 for a review). However, the separate consideration of adiabatic dynamics and nonadiabatic transitions is of limited use for reactions in condensed phase. First, in the case of a multidimensional reaction coordinate the extent of the nonadiabaticity region may sometimes be difficult to determine. Such a situation, for instance, appears if an avoided crossing is highly thermally activated, while the pathways around it with less efficient nonadiabatic transitions may, nevertheless, play a dominant role due to their much more favorable statistical weight. Second, the concept of a single-passage transition probability requires that the velocity autocorrelation decays slowly in the region of nonadiabaticity. If this is not the case, the overall transition probability cannot be resolved into a sequence of the single-passage events. In particular, a solvent-induced damping of motion along the reaction coordinates strongly affects the velocity autocorrelation, which becomes zero in the limiting case of continuous diffusion. Third, the nonadiabaticity region itself can be broad, and involve multiple avoided crossings. Last but not least, quantum interference effects can directly manifest themselves in the reaction dynamics, such that the description of nonadiabatic processes in terms of transition probabilities may not be adequate.

Several alternative theoretical approaches were proposed to overcome some of these problems. A mean-field approximation $^{21-24}$ is capable of a fully dynamic treatment of nonadiabatic transitions, but fails in the regions of large splitting between the PESs. On the other hand, a surface hopping $^{24-27}$ (SH) scheme, based on a "fewest switching" algorithm, provides correct asymptotical solutions and at- 
tempts to resolve the first three problems by letting the trajectory hop between PESs everywhere, in accordance with an ad hoc procedure. The SH scheme is computationally efficient, and nowadays the standard approach to study molecular nonadiabatic reactions, despite of its unknown accuracy, applicability limits, and some other intrinsic drawbacks such as neglect of quantum interference effects ${ }^{28}$ and problems with classically forbidden transitions. ${ }^{29}$

We also mention more general methodologies based on the semiclassical considerations ${ }^{30,31}$ and density matrix formalisms. The former are currently under intensive development, but still rather expensive computationally when applied to realistic simulations in condensed phase. The density matrix approaches, in form of Redfield theory ${ }^{32,33}$ and the quantum-classical Liouville equation ${ }^{34-37}$ (QCLE), were also extensively applied to study nonadiabatic transitions in model systems. ${ }^{38-47}$ Since Redfield theory treats the reaction coordinates quantum mechanically, it is mainly useful for one-dimensional PESs, while the QCLE suffers from the internal inconsistency, ${ }^{48}$ especially when applied to nonadiabatic transitions. ${ }^{49,50}$

Our goal is to formulate alternative approaches to describe nonadiabatic transitions in condensed phase, which should have well defined applicability limits, be capable of dynamic treatment of the transitions with a full account for detailed balance, quantum interference, and memory effects, and with a computation efficiency at least comparable to those of the SH scheme. Motivated by a continued success of transition state theory, ${ }^{4,51,52}$ and its nonadiabatic extensions, ${ }^{53-56}$ for modeling intramolecular reactions under a variety of experimental conditions, we start with a relatively simple situation, assuming fast equilibration in phase space to the temperature of the surrounding media. In this case the reaction coordinates can be considered as belonging to the thermal bath, which will be treated by means of classical mechanics. The latter assumption is reasonable when the distance between the quantum energy levels is less than the mean thermal energy. For a molecule in solution at room temperature this condition is met for almost all degrees of freedom, except for the high-frequency stretch vibrations.

In the preceding paper ${ }^{57}$ we generalized a recently suggested non-Markovian quantum-classical approximation ${ }^{58}$ for the purposes of reaction dynamics in condensed phase. The developed method meets the qualifying standards, it can easily be applied to realistic multidimensional reaction coordinates through the use of an ab initio molecular dynamics (MD) technique, and serves as the basis for the subsequent consideration.

The outline of the paper is as follows. Basic assumptions of the model and the corresponding kinetic equations are presented in Sec. II. An efficient way for the direct solution of these equations in the time domain, based on the MD technique, is formulated in Sec. III. For the model study we employ a Brownian dynamics approach to simulate nuclear dynamics on the PESs involved, as it is briefly described in Appendix B. The simple model of two coupled harmonic oscillators with different curvatures is described in Sec. IV, and serves as the basis for the comparison of the developed approach with known analytic results. The capability of de- scribing various regimes of the nonadiabatic reaction is demonstrated in Sec. V. In the Conclusion we summarize the results obtained.

\section{BASIC ASSUMPTIONS AND KINETIC EQUATIONS}

First of all, it is necessary to define zero-order solution to the problem, or PESs. They are formally specified using a partition of the reacting subsystems on the groups of states, so that the transitions between them mean the reaction of interest. For the distinctness we speak about the transitions between different electron states, although the nature of the reacting subsystem may be rather arbitrary. Then, the Hamiltonians of the PESs are defined as

$$
\hat{F}_{n}=\hat{P}^{n}\left(\hat{H}+\hat{W}+\hat{F}_{0}\right) \hat{P}^{n},
$$

where $\hat{P}^{n}$ is the projector on a given electron state, $\hat{H}$ and $\hat{F}_{0}$ are the Hamiltonians of the reacting subsystem and of the bath, respectively, while $\hat{W}$ is the Hamiltonian of the coupling between them. As pointed out in the Introduction, we assume a classical mechanics description of the dynamics along the reaction coordinates, and fast equilibration to the temperature of the surrounding $T$ media as compared to the time scale of the reaction. The last assumption restricts the class of tractable systems to those, where the motion in the phase space of the reacting subsystem is localized (examples are intramolecular electron/proton transfer, photoisomerization, etc.). Thus, for each electronic state the corresponding PES is characterized by the Hamiltonian function dependent both on the phase space coordinates of the reacting subsystem $Q$ and of the bath $q_{b}$, i.e.,

$$
F_{n}(q)=H_{n}(Q)+W_{n}(q)+F_{0 n}\left(q_{b}\right),
$$

where $q=\left(Q, q_{b}\right)$, and all Hamiltonian functions are specific to the given electronic state.

Note that the projected coupling to the solvent $W_{n}\left(Q, q_{b}\right)$ does not lead to the transitions between distinct PESs, and is assumed to provide an efficient equilibration, so that the distribution in the phase space of $n$th PES is $\sim \exp \left[-F_{n}(q) / k_{B} T\right]$, where $k_{B}$ is the Boltzmann constant. Accordingly, the state of the system is fully determined by the set of probabilities $\sigma_{n}(t)$ to be on the corresponding multidimensional PES, defined by Eq. (2).

The operators of the couplings between PESs are specified as

$$
\hat{W}_{n k}=\hat{P}^{n} \hat{W} \hat{P}^{k}, \quad \hat{W}_{k n}=\hat{W}_{n k}^{\dagger},
$$

where the dagger superscript denotes Hermitian conjugate. In the absence of quantum-mechanical tunneling, and in the classical limit they are reduced to the set of couplings

$$
W_{n k}(q)=W_{k n}^{*}(q),
$$

where the asterisk denotes the complex conjugate. The theory ${ }^{57}$ allows an intermediate strength of these couplings under condition 


$$
\frac{\left|W_{n k}(q)\right| \tau_{b}}{\hbar} \ll 1, \quad \tau_{b}=\frac{\hbar}{\pi k_{B} T},
$$

where $\hbar$ is the Planck constant and $\tau_{b}$ is a lifetime of the quantum correlations due to an energy dispersion (per degree of freedom) of the canonical bath, see Refs. 57 and 58 for more details. The partition of the system (2) and (4) is rather formal, but the assumption about time scale separation of the equilibration and of the reaction makes the approach to be more appropriate for the description of nonadiabatic reactions.

Recently ${ }^{59}$ on the basis of the QCLE approach it was found that increasing the strength of the coupling the true adiabatic limit cannot be reached for diffusive (thermally damped) motion through the intersection point. This result, however, has to be considered with care. Apart from other inconsistencies, the QCLE completely ignores the existence of the quantum component of the nonequilibrium energy flux between the PESs. The latter becomes dominant at $\left|\hat{W}_{n k}(q)\right| \tau_{b} / \hbar \geq 1$, see also Ref. 60 , and the true adiabaticity is reached in this limit even at diffusive motion. On the other hand, the small parameter (5) of the theory does not coincide with the familiar Massey parameter, which is used to distinguish between adiabatic and nonadiabatic regimes in the absence of a thermal bath. Thus, Eq. (5) may serve as the criterion of the nonadiabaticity of thermally damped systems and solvent-induced transitions.

In the following we set $\hbar=k_{B}=1$ to write formulas in a more compact form. Then the potentials, the nonadiabatic couplings, and the temperature are measured in $\mathrm{rad} / \mathrm{s}$. In these units $1 \mathrm{eV}=1.52 \times 10^{15} \mathrm{rad} / \mathrm{s}$ and $1 \mathrm{~K}=k_{B} / \hbar=1.33$ $\times 10^{11} \mathrm{rad} / \mathrm{s}$.

The kinetic equations for the model under consideration are derived from the more general ones, obtained in the preceding paper. In our case the quantum subsystem for each reaction channel consists of a single state, while the associated equilibrium bath is characterized by the Hamiltonian (2). Then, extending the results ${ }^{57}$ to the case of multiple PESs, we obtain the following kinetic equations for the probabilities $\sigma_{n}(t)$ to be on the corresponding PES:

$$
\frac{d}{d t} \sigma_{n}(t)=-\sum_{\substack{k=1 \\ k \neq n}}^{N} \int \operatorname{Im}\left[\left(\chi_{n k}^{(n)}(q, t)+\chi_{n k}^{(k)}(q, t)\right) W_{k n}(q)\right] d q,
$$

where $N$ is the number of distinct PESs, the couplings between them were defined earlier, and the auxiliary functions, which contain information about memory, obey

$$
\begin{aligned}
\frac{\partial \chi_{n k}^{(n)}(q, t)}{\partial t}= & \gamma_{n k}(q, t) \psi^{(n)}(q)-i \Omega_{n k}(q) \chi_{n k}^{(n)}(q, t) \\
& +\mathcal{L}_{q}^{(n)} \chi_{n k}^{(n)}(q, t)
\end{aligned}
$$

where

$$
\psi^{(n)}(q)=e^{-F_{n}(q) / T} / \mathcal{Z}_{n}, \quad \mathcal{Z}_{n}=\int e^{-F_{n}(q) / T} d q
$$

is the static contour for the $n$th reaction channel (normalized Boltzmann distribution), and the source term

$$
\begin{aligned}
\gamma_{n k}(q, t)= & i W_{n k}(q)\left(\sigma_{n}(t)-\sigma_{k}(t)\right) \\
& +\left(U_{n k}(q)+Y_{n k}(q) \frac{d}{d t}\right)\left[\sigma_{n}(t)+\sigma_{k}(t)\right] .
\end{aligned}
$$

Equation for $\chi_{n k}^{(k)}(q, t)$ is obtained from Eq. (7) replacing the superscript $n$ with $k$. The difference between $\chi_{n k}^{(n)}(q, t)$ and $\chi_{n k}^{(k)}(q, t)$ lies in distinct PESs ( $n$th or $k$ th), along which the dynamics are simulated. The latter is specified by the linear functional operators $\left(\mathcal{L}_{q}^{(n)}\right.$ or $\left.\mathcal{L}_{q}^{(k)}\right)$, defining the motion in the phase space of the corresponding PES. Here also

$$
\Omega_{n k}(q)=F_{n}(q)-F_{k}(q)
$$

is the difference of the Hamiltonian functions (2) of $n$th and $k$ th PES, while

$$
U_{n k}(q)=i \tanh \left(\frac{\epsilon_{n k}}{2 T}\right) W_{n k}(q),
$$

and

$$
Y_{n k}(q)=\frac{1}{2 T} \frac{W_{n k}(q)}{\cosh ^{2}\left(\frac{\epsilon_{n k}}{2 T}\right)},
$$

where

$$
\epsilon_{n k}=-T \ln \left(Z_{n} / Z_{k}\right)
$$

is the change of free energy due to the transition from $n$th to $k$ th PES, expressed in terms of the statistical sums of the corresponding PESs. Two recipes can be used for the calculation of $Z_{i}$, a classical one [apart from a prefactor, which anyway does not play role in Eq. (13), the classical partition function is specified by Eq. (8)], or a quantum-mechanical recipe. The latter accounts the zero-point energy effects, but may be prohibitively expensive for multidimensional PESs.

The initial state of the system is specified by the set of initial probabilities $\sigma_{k}(0)$, and by the nonzero initial conditions for the auxiliary functions

$$
\chi_{n k}^{(n, k)}(q ; t=0)=Y_{n k}(q) \psi^{(n)}(q)\left(\sigma_{n}(0)+\sigma_{k}(0)\right),
$$

which appear due to the formation of specific correlations between the quantum subsystem and the bath on the time scale $\tau_{b}$, see Refs. 57 and 58 for more details. Finally, interchanging the two subscripts of the auxiliary function leads to the complex conjugate, i.e., as it follows from Eqs. (4) and (7)-(12), one has

$$
\chi_{k n}^{(n, k)}(q, t)=\left[\chi_{n k}^{(n, k)}(q, t)\right]^{*},
$$

which allows to avoid unnecessary calculations.

The kinetic equation (6) preserves the total probability, as may be readily proved by taking the sum over all PESs, which gives $d \Sigma_{n}^{N} \sigma_{n}(t) / d t=0$. The stationary solution of Eqs. (6) and (7) is readily found from the conditions 


$$
i W_{n k}(q)\left(\sigma_{n}-\sigma_{k}\right)=-U_{n k}(q)\left(\sigma_{n}+\sigma_{k}\right),
$$

which means the absence of the source term on the righthand side of Eq. (7), and gives

$$
\sigma_{n} / \sigma_{k}=e^{-\epsilon_{n k} / T}=Z_{n} / Z_{k} .
$$

Thus, the stationary solution satisfies detailed balance with respect to the change of the free energy in the course of reaction.

Equation (7) on the right-hand side contains the difference of the bath Hamiltonians $\Omega_{n k}(q)$ defined by Eq. (10). This term was absent ${ }^{58}$ with bath dynamics independent of the state of the quantum subsystem. It plays, however, a key role for the model being considered, as it localizes the transitions to the PES crossing regions. If the difference of the PES Hamiltonians is large, it produces fast oscillations in the auxiliary functions, which considerably reduce contributions from these unfavorable areas. Note that we mean the crossing in phase space, rather than in position space. In particular, when the effective masses for the motion along distinct PESs are different, the change of the kinetic energy influences the location of the transition region as well.

We would also emphasize that Eqs. (6) and (7) do not involve any mean-field approximation, which is known to be a source of severe problems, especially when applied to nonadiabatic transitions. Instead of the mean-field treatment, Eqs. (6) and (7) assert a "mean-evolution" recipe, when the auxiliary functions are propagated along the given PESs, then summing up contributions, but do not evolve in a meanfield potential.

\section{MOLECULAR DYNAMICS APPROACH}

A numerical solution of Eqs. (6) and (7) presents considerable difficulty in view of the partial derivatives in Eq. (7). This problem can, however, be overcome by introducing a MD-based algorithm similar to that proposed in Ref. 48. For ease of presentation we restrict ourselves to just two PESs, but the generalization of the method is straightforward.

For a two-PESs case one deals with single coupling $W(q)=W_{12}(q)$ and two auxiliary functions $\chi^{(1,2)}(q, t)$ $=\chi_{12}^{(1,2)}(q, t)$, which allows one to reduce the number of indices. In view of the normalization condition

$$
\sigma_{1}(t)+\sigma_{2}(t)=1,
$$

it is convenient to work with the population difference, defined as

$$
\alpha(t)=\frac{1}{2}\left(\sigma_{1}(t)-\sigma_{2}(t)\right),
$$

with the inverse relations

$$
\sigma_{1}(t)=\frac{1}{2}+\alpha(t), \quad \sigma_{2}(t)=\frac{1}{2}-\alpha(t) .
$$

Then we replace the phase space dynamics by the MD averages, and obtain from Eqs. (6) and (19)

$$
\begin{aligned}
\frac{d \alpha(t)}{d t}= & -\frac{1}{L} \sum_{l=1}^{L} \operatorname{Im}\left(W_{l}^{*(1)}(t) \chi_{l}^{(1)}(t)\right) \\
& -\frac{1}{M_{m}} \sum_{m=1}^{M} \operatorname{Im}\left(W_{m}^{*(2)}(t) \chi_{m}^{(2)}(t)\right),
\end{aligned}
$$

where $L$ and $M$ in practice are large numbers of multidimensional MD trajectories on the first and second PES, respectively, which are assumed to be sufficient to explore the phase spaces, while the nonadiabatic couplings parametrically depend on time along the given MD trajectory. Here the superscript labels the PES to which the trajectory belongs, while the subscript indicates a particular MD trajectory, taken from the corresponding equilibrium simulation. In turn, the sets of auxiliary functions satisfy

$$
\frac{d}{d t} \chi_{l}^{(1)}(t)=i W_{l}^{(1)}(t)\left[2 \alpha(t)+\tanh \left(\frac{\epsilon}{2 T}\right)\right]-i \Omega_{l}^{(1)}(t) \chi_{l}^{(1)}(t),
$$

and

$$
\frac{d}{d t} \chi_{m}^{(2)}(t)=i W_{m}^{(2)}(t)\left[2 \alpha(t)+\tanh \left(\frac{\epsilon}{2 T}\right)\right]-i \Omega_{m}^{(2)}(t) \chi_{m}^{(2)}(t),
$$

with the "slipped" initial conditions

$$
\chi_{l}^{(1)}(0)=\frac{1}{2 T} \frac{W_{l}^{(1)}(0)}{\cosh ^{2}\left(\frac{\epsilon}{2 T}\right)}, \quad \chi_{m}^{(2)}(0)=\frac{1}{2 T} \frac{W_{m}^{(2)}(0)}{\cosh ^{2}\left(\frac{\epsilon}{2 T}\right)} .
$$

Note that the term containing the time derivative on the right-hand side of Eq. (9) is absent in Eqs. (22) and (23) in view of the normalization condition (18), but appears for the case of three and more PESs. Here also

$$
\epsilon=\epsilon_{12}=-T \ln \left(Z_{1} / Z_{2}\right),
$$

and the difference of the bath Hamiltonians

$$
\Omega(q)=F_{1}(q)-F_{2}(q)
$$

is calculated along the same trajectory either evolving on the first or on the second PES.

In our MD simulations we used an algorithm of the leapfrog type, where the trajectory is represented as a series of switching positions in phase space of multidimensional PES (2), and the time between switches is equal to the time step $\Delta t$. The numerical approach to integrate Eqs. (21)-(23) is described in Appendix A. We emphasize that the MD trajectories can be simulated by any appropriate algorithm, and that the PESs are inherently multidimensional.

At the end of this section we discuss the numerical efficiency of the developed approach, which we would abbreviate as SANT (statistical approach to the nonadiabatic transitions), in comparison to the popular surface hopping scheme. The calculation of the MD trajectories is the most expensive part in both methods. The principal difference between them is that instead of single hopping trajectory calculations with 


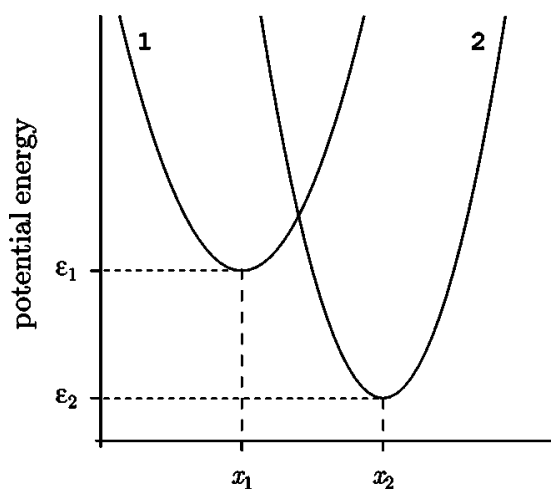

FIG. 1. Schematic representation of the potential energy surfaces. Equilibrium positions are denoted as $x_{1,2}$, while $\varepsilon_{1,2}$ are vertical displacements.

subsequent averaging of the kinetics in the framework of the $\mathrm{SH}$ scheme, we need sets of equilibrium MD trajectories along the PESs involved without hops. The sufficient number of these equilibrium trajectories is difficult to estimate a priori, but it should be comparable to the number of the $\mathrm{SH}$ trajectories, necessary to obtain a convergent result. The equilibrium trajectories in our method can be calculated in advance and stored for subsequent use. Thus, one can conclude that both the SH scheme and SANT have comparable computational cost. However, if a series of calculations with different values of the nonadiabatic couplings is requested, which is often the case when fitting experimental data, then SANT becomes more efficient, since it allows the same set of the MD trajectories to be used, while the $\mathrm{SH}$ scheme requires a complete recalculation of the hopping trajectories.

Although the SANT can be applied to ab initio modeling of many realistic systems, provided that the PESs of interest and the nonadiabatic couplings are available, ${ }^{61}$ we restrict ourselves to a simple model of two coupled one-dimensional harmonic oscillators, where a solvent influence is accounted for via phenomenological Brownian dynamics approach. We do not issue here the challenge to test the SANT against more sophisticated benchmark models, which are frequently used to get an impression about the usefulness of various hybrid schemes. This is because the underlying theory ${ }^{58,57}$ was developed as a rigorous expansion on the known small parameter, and the applicability limits are known explicitly. Instead of extensive benchmarking, we prefer to concentrate on distinctive features of the SANT, and the use of a simple model makes the discussion more transparent.

\section{TWO COUPLED ONE-DIMENSIONAL HARMONIC OSCILLATORS}

To demonstrate the capabilities of SANT, and to check its accuracy against known analytical solutions, we apply it to a simple model of two coupled harmonic oscillators, schematically shown in Fig. 1.

Fairly often, nonadiabatic transitions considerably change the properties of a molecule. The new normal modes may have different effective masses, and the change of equilibrium bond lengths affects the frequencies of the normal modes as well. To account for this fact in our model, we assume different masses $\left(\mu_{1,2}\right)$ and frequencies $\left(\omega_{1,2}\right)$ for the first and the second PESs, so that their Hamiltonians take the form

$$
\begin{aligned}
& H_{1}(p, x)=\varepsilon_{1}+\frac{p^{2}}{2 \mu_{1}}+\frac{\mu_{1} \omega_{1}^{2}\left(x-x_{1}\right)^{2}}{2}, \\
& H_{2}(p, x)=\varepsilon_{2}+\frac{p^{2}}{2 \mu_{2}}+\frac{\mu_{2} \omega_{2}^{2}\left(x-x_{2}\right)^{2}}{2},
\end{aligned}
$$

where $\varepsilon_{1,2}$ are the vertical displacements while $x_{1,2}$ are the equilibrium positions of the corresponding oscillators. The coupling to the surrounding media, necessary for fast equilibration in phase space, is accounted phenomenologically by a Brownian dynamics model, ${ }^{62}$ which treats solvent effects by means of the friction coefficients $\xi_{1,2}$, related to the diffusion coefficient $D$ via Einstein's relation

$$
\xi_{1,2}=T /\left(\mu_{1,2} D\right) \text {. }
$$

In this case the multidimensional trajectories on the PESs (2) are reduced to the trajectories in the phase space of harmonic oscillators (27) and (28), but with the friction. The details of the algorithm, used to generate these MD trajectories, are given in Appendix B.

Before propagation in time, the free-energy difference (25) has to be specified. It may be estimated from experimental data or calculated by appropriate method. ${ }^{63,64}$ This is a nontrivial task in practical applications, but we face no difficulties for the simple model under consideration, where the statistical sums can easily be found using the quantummechanical recipe

$$
Z=\sum_{i} e^{-\mathcal{E}_{i} / T}
$$

where the sum is taken over all eigenstates of the PES, and $\mathcal{E}_{i}$ are the corresponding eigenvalues, or the classicalmechanical one, when the summation is replaced by the integration over phase space, i.e.,

$$
Z=\frac{1}{2 \pi} \iint e^{-H(p, x) / T} d p d x .
$$

[Note that the multiplier $1 /(2 \pi)$ in Eq. (31) appears as a consequence of the prefactor $1 / h$ in the definition of the classical partition function, and of using the frequency units $(\hbar=1)$.]

The use of the quantum-mechanical recipe (30) properly accounts for the zero-point energy in detailed balance. On the other hand, diagonalization of the PES Hamiltonian becomes prohibitively expensive for the multidimensional case, where the classical-mechanical recipe (31) is of practical importance, especially for low-frequency reaction coordinates. Among other reasons, neglect of the zero-point energy in the standard classical mechanics approaches to the reaction dynamics gave rise to more systematic but at the same time more computationally expensive treatments. SANT is even more flexible; it can easily be extended to treat the dynamics of high-frequency reaction coordinates quantum mechanically. 
For the one-dimensional harmonic oscillator with the eigenvalues

$$
\mathcal{E}_{n}=\varepsilon+\omega\left(n+\frac{1}{2}\right), \quad n=0,1, \ldots
$$

the quantum statistical sum is

$$
Z_{\mathrm{q}}=\frac{e^{-(\varepsilon+\omega / 2) / T}}{1-e^{-\omega / T}} \underset{\omega \ll T}{\simeq} \frac{T e^{-\varepsilon / T}}{\omega},
$$

while the classical partition function takes the form

$$
Z_{\mathrm{cl}}=\frac{T e^{-\varepsilon / T}}{\omega} .
$$

As it should be, the quantum statistical sum and the classical partition function coincide, when the splittings between the energy levels become much less than the mean thermal energy $T$. For the case of equal curvatures of the PES both recipes simply give $\epsilon=\Delta \varepsilon$, where

$$
\Delta \varepsilon=\varepsilon_{1}-\varepsilon_{2},
$$

i.e., the change of the free energy is simply the difference of the vertical displacements. However, in the situation of considerably different PES curvatures, the zero-point energy effects may play an important role, and the use of the quantum-mechanical recipe (33) is preferable.

\section{RESULTS AND DISCUSSION}

We start with the case of equal oscillator masses, frequencies, and frictions for both PESs, and assume $T$ $=300 \mathrm{~K}, \omega_{1}=\omega_{2}=\omega=0.03 \mathrm{eV}, \mu_{1}=\mu_{2}=\mu=72 \mathrm{amu}$ (mass of six carbon atoms), $x_{2}-x_{1}=0.1 \AA$. This corresponds to a reorganization energy of $\simeq 0.08 \mathrm{eV}$, which is typical for the initial charge separation reaction in photosynthetic bacterial reaction centers. ${ }^{65}$ The friction is assumed to be controlled by the solvent viscosity. For a diffusion coefficient $D$ $=10^{-6} \mathrm{~cm}^{2} / \mathrm{s}$, Eq. (29) gives $\bar{\xi}=\xi / \omega=7.6$, and for the simulations this parameter has mainly been taken from the range $1-10$, although the spread of diffusion coefficients may be very large, with the environment ranging from supercritical fluids to biological membranes. Energy bias $\Delta \varepsilon$ and nonadiabatic coupling strength, assumed to be coordinate independent, have been varied. The MD simulations involved 1000 trajectories for each PES, several such calculations were averaged to improve the accuracy.

In many situations, the kinetics of electron transfer is well described by the rates of forward $k_{f}$ and backward reactions $k_{b}$. Assuming the validity of the rate regime, one has

$$
\alpha(t)=\alpha_{\infty}+\left[\alpha(0)-\alpha_{\infty}\right] e^{-\left(k_{f}+k_{b}\right) t},
$$

we also used this expression to compare with the results of our simulations. For the case of equal parameters of the bath oscillators, these rates are of the form ${ }^{6,67}\left(\hbar=k_{B}=1\right)$

$$
k_{f}=W^{2} \sqrt{\frac{\pi}{\lambda T}} \exp \left(-\frac{(\epsilon-\lambda)^{2}}{4 \lambda T}\right), \quad \lambda=\frac{\mu \omega^{2}\left(x_{2}-x_{1}\right)^{2}}{2},
$$

while $k_{b}$ is related to $k_{f}$ via the detailed balance

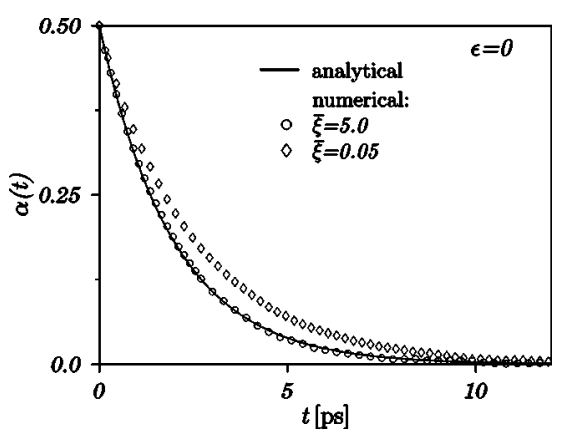

FIG. 2. Kinetics of electron transfer at zero energy bias, weak coupling $\left(W=3 \times 10^{-3} \mathrm{eV}\right)$, and different frictions, indicated in the figure. The analytic result was calculated by Eqs. (36)-(38). Slowing down relaxation of the bath modes $(\bar{\xi} \rightarrow 0)$ causes thermal activation to be the limiting step of the reaction.

$$
k_{b}=k_{f} e^{-\epsilon / T},
$$

and the change of the free energy is equal to the energy bias, i.e., $\epsilon=\Delta \varepsilon$.

Figure 2 shows the kinetics of electron transfer at zero energy bias and relatively weak nonadiabatic coupling $W$ $=3 \times 10^{-3} \mathrm{eV}$. The initial condition $\alpha(0)=0.5$ corresponds to the absence of population of the second state, while the reaction proceeds towards equilibration of the states $\left(\alpha_{\infty}=0\right)$. It is readily seen that analytical expressions (36)-(38) accurately describe this situation, except for the low friction limit. In the latter case the energy flow within the PES slows down, and thermal activation becomes the limiting step of the reaction. Note, however, that we assumed canonical equilibrium for each PES in the course of reaction, which, naturally, is violated as $\xi \rightarrow 0$. None the less, our approach properly reflects the general trend of the reaction rate in the low friction limit, which is accounted for perturbatively.

Then we consider the case of an activationless reaction, when the energy bias is equal to the reorganization energy, $\epsilon=0.078 \mathrm{eV}$, see Fig. 3. Two different initial conditions were used, $\alpha(0)=0.5$ and $\alpha(0)=-0.5$. In the first case, the reaction starts from the state with higher energy, and the population difference changes its sign in the course of time, while start-

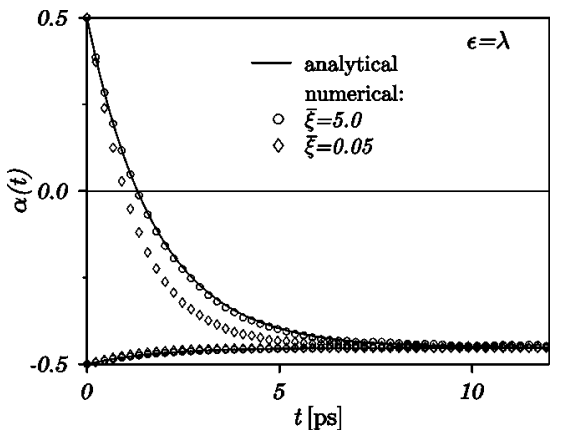

FIG. 3. Kinetics of electron transfer at energy bias equal to the reorganization energy $(\epsilon=0.078 \mathrm{eV})$, weak coupling $\left(W=3 \times 10^{-3} \mathrm{eV}\right)$, and different frictions, indicated in the figure. Two different initial conditions, corresponding to the start from upper $[\alpha(0)=0.5]$ and lower $[\alpha(0)=-0.5]$ states, were used. The analytic result was calculated by Eqs. (36)-(38). In the case of the activationless reaction, decreasing the friction $(\xi \rightarrow 0)$ increases the number of passages through an intersection point per unit time, which accelerates the reaction. 


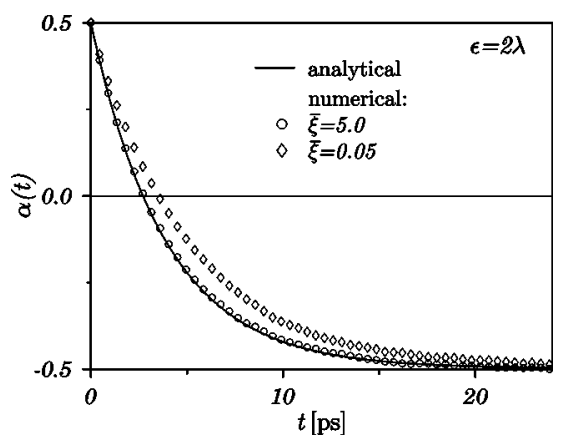

FIG. 4. Kinetics of electron transfer at energy bias $\epsilon=2 \lambda=0.155 \mathrm{eV}$, weak coupling $\left(W=3 \times 10^{-3} \mathrm{eV}\right)$, and different frictions indicated in the figure. The reaction has twice as long a time scale, than for $\epsilon=0$, while decreasing the friction results in transitions being controlled by thermal activation.

ing from the lower lying PES we observe small changes in population difference, since the initial state is already pretty close to the stationary Boltzmann distribution. Once again, the numerical simulation at $\bar{\xi}=5$ perfectly matches analytical result, while in the low friction limit the reaction accelerates, unlike the case considered above. This is due to the activationless character of the electron transfer at the parameter values used. Decreasing the friction increases the number of passages through the intersection point per unit time, and, therefore, accelerates the reaction. This is a purely dynamical effect of the friction.

Increasing the energy bias to $\epsilon=2 \lambda=0.155 \mathrm{eV}$ we arrive at the same activation energy, but the crossing now occurs in the so-called inverted region. The kinetics for this case is shown in Fig. 4. The equilibrium population of upper PES is very small, and the time scale of the reaction is two times larger than in Fig. 2. The rates of the forward transfer at $\epsilon=0$ and $\epsilon=2 \lambda$ are equal to each other, as it follows from the analytical expression (37), but the rate of backward transfer satisfies $k_{b}=k_{f}$ at $\epsilon=0$, while being $k_{b} \ll k_{f}$ at $\epsilon=2 \lambda$. This explains the difference in the relaxation time scales, see Eq. (36). Again, at low frictions the reaction becomes controlled by thermal activation, similarly to the case of $\epsilon=0$.

In the above situations, the kinetics of the reaction was exponential, while SANT correctly reproduced the freeenergy-gap law and the general trends of the reaction rate in the low friction limit. Besides, SANT is valid beyond the rate description of the nonadiabatic transitions, and fully accounts for the quantum coherence effects in the kinetics of the reaction. To demonstrate this, we considerably reduce the reorganization energy to $\lambda=0.016 \mathrm{eV}$, and increase the strength of the nonadiabatic coupling to $W=0.02 \mathrm{eV}$. We also assume $\epsilon=0.02 \mathrm{eV}$ and $\bar{\xi}=5$. The kinetics in this case exhibits strong transient oscillations and is shown in Fig. 5. Two types of the initial conditions for the auxiliary functions $\chi^{(1,2)}$ were used. In the first case we neglected specific cross correlations between the quantum subsystem and the canonical bath created during the initial time period $\left(t \lesssim \tau_{b}\right)$, which corresponds to zero initial values of $\chi^{(1,2)}$. The corresponding kinetics is shown in Fig. 5 by the dashed line. In the second case proper initial conditions (24) were used, and the kinetics for this case is plotted in Fig. 5 as the solid line. The values

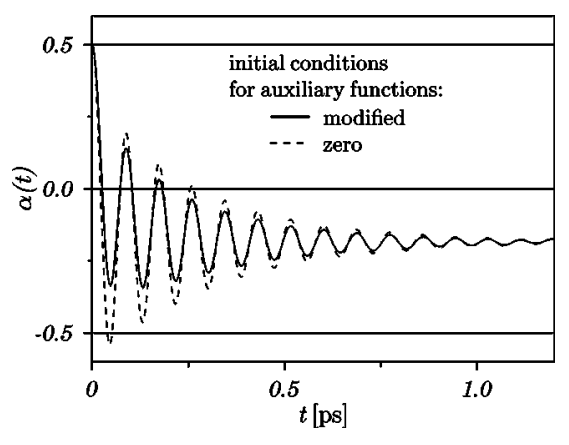

FIG. 5. Kinetics of electron transfer beyond the rate regime. The initial condition for the population difference $\alpha(0)=0.5$, while those for auxiliary functions are either set to zero (dashed line) or taken from Eq. (24) (solid line). The parameters used are $\epsilon=0.02 \mathrm{eV}, \lambda=7.8 \times 10^{-4} \mathrm{eV}, W=0.02 \mathrm{eV}$, and $\bar{\xi}=5$. It is seen that properly accounting for the correlations created during the early time evolution $\left(t \lesssim \tau_{b}\right)$ is indeed important to preserve the positivity within the applicability limits.

of $|\alpha(t)|>0.5$ indicate negative probabilities, while the use of proper initial conditions for the auxiliary functions removes this unphysical behavior.

A generalization of the Marcus-Levich-Dogonadze nonadiabatic rate constants (37) and (38) to the case of different curvatures was suggested in Refs. 68 and 69. This corresponds to different reorganization energies for the forward and backward electron transfer, a feature also observed experimentally. ${ }^{65}$ In this case, unlike the situation of equal curvatures of the bath oscillators, the change of free energy due to the reaction is not equal to the energy bias, and there may be two or no crosspoints. First of all, we consider a situation when the PESs do cross, so that the transitions can be considered as localized at crosspoints, and the corresponding analytical results are of relatively simple form.

Figure 6 shows the kinetics of the reaction at different reorganization energies for the forward and backward transfer, $\lambda_{1}=0.078 \mathrm{eV}$, and $\lambda_{2}=0.012 \mathrm{eV}$, respectively. Other parameters are as follows: $T=300 \mathrm{~K}, \bar{\xi}_{1}=\bar{\xi}_{2}=10$, while the nonadiabatic coupling strength $W=3 \times 10^{-3} \mathrm{eV}$. Energy bias is varied and take the following values: $\Delta \varepsilon=0.01 \mathrm{eV}$ (circles) and $\Delta \varepsilon=0$ (squares). The corresponding analytic results ${ }^{68,69}$ are plotted as solid lines. [Note that the definition of the nonadiabatic coupling strength in our case differs by a factor of 2 , so that the prefactor of $1 / 4$ in the formulas in

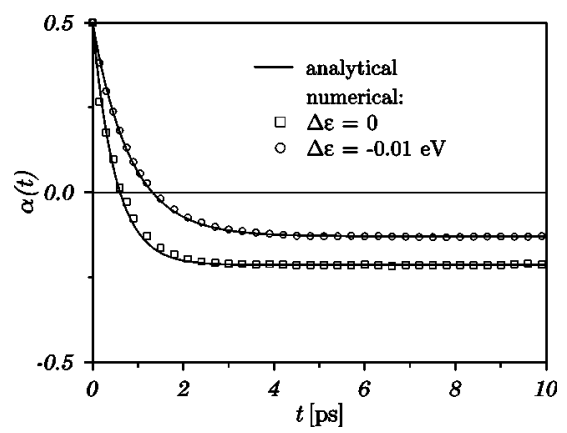

FIG. 6. Kinetics of the nonadiabatic transitions for the case of different curvatures of PES oscillators. The parameters are $T=300 \mathrm{~K}, \lambda_{1}=0.078 \mathrm{eV}$, $\lambda_{2}=0.012 \mathrm{eV}, \bar{\xi}_{1}=\bar{\xi}_{2}=10, W=3 \times 10^{-3} \mathrm{eV}$, and $\alpha(0)=0.5$. Calculations were done for two different energy biases $\Delta \varepsilon=-0.01 \mathrm{eV}$ (circles) and $\Delta \varepsilon=0$ (squares). Analytical results were calculated by formula taken from Ref. 69. 


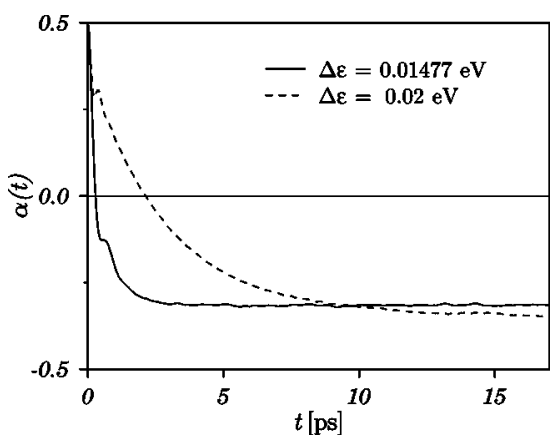

FIG. 7. Same as for Fig. 6 but at larger energy bias. At $\Delta \varepsilon=0.01477 \mathrm{eV}$ (solid line) the potentials touch each other, while at $\Delta \varepsilon=0.02 \mathrm{eV}$ (dashed line) there are no crossing points. The results were calculated numerically by the suggested approach; in the former case the kinetics is considerably accelerated (reaction rates in contact approximation diverge), while in the latter case the kinetics slow down again as the energy gap (in the inverted region) between the PESs increases.

Refs. 68 and 69 for the rate constants should be dropped.] Finally, the statistical sums of the baths were calculated by the classical recipe (34) for compatibility purposes, although accurate (quantum) canonical equilibrium can be incorporated in our approach by using Eq. (33). At $\Delta \varepsilon=0$, the stationary value of the population difference is not equal to 0.5 , while for $\Delta \varepsilon=-0.01 \mathrm{eV}$ the transfer proceeds even against the energy bias. This is because for the given values of $\Delta \varepsilon$ the enthropic part of the reaction free energy dominates.

It is readily seen that SANT accurately reproduces analytic results for different curvatures of the PES oscillators. However, the accuracy of analytic formulae, obtained in a "contact" approximation (assuming the transitions to be localized at crosspoints), degrades when increasing energy bias. Finally, at $\Delta \varepsilon \simeq 0.015 \mathrm{eV}$ the PESs only touch each other, while analytic expressions for the rate constants in the contact approximation diverge. Although Ref. 68 provides us with the analytical formulae even for the case of noncrossing PESs, these expressions are rather cumbersome and were not used for the comparison. Besides, we would like to emphasize that the QCLE-predicted ${ }^{68}$ deviation of the stationary distribution in the case of noncrossing PESs from those expected from the statistical thermodynamical consideration cannot be obtained from our approach. This is because the relaxation to the correct stationary value is an intrinsic property of the kinetic equations used, see Sec. II.

Figure 7 shows the kinetics of the reaction for the same parameter values as on Fig. 6, but at larger energy bias. When approaching the value $\Delta \varepsilon=0.01477 \mathrm{eV}$, the two crossing points move close to each other. The contact approximation predicts acceleration of the kinetics, but at the same time it becomes less applicable. Finally, at $\Delta \varepsilon$ $>0.01477 \mathrm{eV}$ there are no crosspoints, and the transition region delocalizes. The kinetics is indeed fastest at $\Delta \varepsilon$ $=0.01477 \mathrm{eV}$, then slows down when further increasing energy bias, since the energy gap between the PESs becomes larger.

\section{CONCLUSION}

We presented a new statistical approach to nonadiabatic transitions. It uses a classical mechanics description of the dynamics along the potential energy surfaces, and requires fast equilibration of the reaction coordinates to the temperature of the surrounding media, such that the reaction does not change significantly the equilibrium distribution in their phase spaces. The developed methodology has known applicability limits, fully accounts for detailed balance, including zero-point energy effects, quantum coherence, arbitrarily long memory, and change of the free energy in the course of reaction. The strength of the nonadiabatic couplings can be varied over a wide range, which allows to model the kinetics of nonadiabatic processes starting from a subpicosecond time scale. In addition, the approach is compatible with any $a b$ initio molecular dynamics method to simulate the dynamics along the reaction coordinates, which can be of multidimensional character, and constitutes a powerful framework for accurate modeling of the nonadiabatic transitions in thermally damped systems.

SANT has several important advantages over existing quantum-classical methodologies. As compared to the nonadiabatic extension of the transition state theory, we removed the restriction of a one-dimensional reaction coordinate, and the phenomenological description of the elementary act via transition probabilities. Besides, our approach is valid beyond the rate regime and is capable of describing quantum interference effects and non-Markovian evolution. In contrast to the quantum-classical Liouville equation approach, SANT does not lead to a mean potential treatment of the transition phases, which is known to be the source of unphysical predictions, and properly handles the cross correlations between the quantum subsystem and the thermal bath.

The molecular dynamics implementation of SANT is also very different from the surface hopping scheme, being of comparable computational cost. First, the problem cannot be reduced to the evolution of the quantum subsystem along single trajectories with subsequent averaging, instead the bath is always represented by a large set of equilibrium trajectories. This reflects the fact that both quantum and classical subsystems are treated on a similar probabilistic footing. Second, the trajectories do not hop between the potential energy surfaces, as a result a fewest switching algorithm and rescaling of the velocities to conserve the total energy are obsolete. Third, the most time consuming molecular dynamics part of the simulation can be done in advance, the same set of trajectories is usable to perform calculations for different strengths of the nonadiabatic couplings.

The enhanced capabilities of SANT were demonstrated, using a simple model of two coupled harmonic oscillators, and a Brownian dynamics approach to simulate the motion along the reaction coordinate. It was shown that SANT precisely reproduces the free-energy-gap law in the rate regime and at moderate friction. An example of the kinetics beyond the rate regime was given, where the proper modification of the initial conditions to account for the early time evolution is of primary importance to preserve the positivity within the applicability limits. However, both in the low and in the high friction limits the transitions are controlled either by diffusion in energy space or by spatial diffusion, and, therefore, the reaction coordinates cannot be treated as belonging to the 
canonical bath. Nonetheless, at small deviations from equilibrium, SANT properly describes the reaction dynamics at low friction as well.

There are many other situations, when the reaction coordinate has to be treated separately from the bath degrees of freedom. Examples are intermolecular electron transfer, association/dissociation reactions, etc. These cases, as well as the intramolecular nonadiabatic transitions over the complete range of frictions, require a more delicate approach. This work is now in progress.

\section{ACKNOWLEDGMENTS}

The author is grateful to Professor Jörg Schroeder for helpful discussions and to Professor Jürgen Troe for his continuous support. This work was partially supported by the Deutsche Forschungsgemeinschaft (DFG).

\section{APPENDIX A: NUMERICAL APPROACH}

In the leap-frog MD simulations the phase space coordinates are assumed to be fixed during the time step $\Delta t$. Accordingly, the time dependent functions on the right-hand sides of Eqs. (21)-(23) take constant values during the time step $\Delta t$, and, therefore, it is sufficient to construct the numerical algorithm for the propagation of the solution on the length of the single time step (from $t=0$ to $t=\Delta t$ ) and with constant coefficients.

First, the following relation holds:

$$
\begin{aligned}
\alpha(\Delta t)-\alpha(0)= & \frac{1}{L} \sum_{l=1}^{L} \operatorname{Im}\left[W_{l}^{*(1)} \frac{\chi_{l}^{(1)}(\Delta t)-\chi_{l}^{(1)}(0)}{i \Omega_{l}^{(1)}}\right] \\
& +\frac{1}{M} \sum_{m=1}^{M} \operatorname{Im}\left[W_{m}^{*(2)} \frac{\chi_{m}^{(2)}(\Delta t)-\chi_{m}^{(2)}(0)}{i \Omega_{m}^{(2)}}\right],
\end{aligned}
$$

which is proved as follows. Introducing the new auxiliary functions (in the "interaction representation") as

$$
\tilde{\chi}_{l}^{(1)}(t)=e^{i \Omega_{l}^{(1)} t} \chi_{l}^{(1)}(t)
$$

which satisfy

$$
\frac{d}{d t} \widetilde{\chi}_{l}^{(1)}(t)=i W_{l}^{(1)} e^{i \Omega_{l}^{(1)} t}\left[2 \alpha(t)+\tanh \left(\frac{\epsilon}{2 T}\right)\right],
$$

we derive (integrating by parts)

$$
\begin{aligned}
& \operatorname{Im}\left[\int_{0}^{\Delta t} W_{l}^{*(1)} \chi_{l}^{(1)}(t) d t\right]=\operatorname{Im}\left[\int_{0}^{\Delta t} W_{l}^{*(1)} e^{-i t} \Omega_{l}^{(1)} \widetilde{\chi}_{l}^{(1)}(t) d t\right] \\
& =-\operatorname{Im}\left[\left.W_{l}^{*(1)} \frac{e^{-i \Omega_{l}^{(1)} t} \tilde{\chi}_{l}^{(1)}(t)}{i \Omega_{l}^{(1)}}\right|_{0} ^{\Delta t}\right. \\
& \left.+\int_{0}^{\Delta t} W_{l}^{*(1)} \frac{e^{-i \Omega_{l}^{(1)} t}}{i \Omega_{l}^{(1)}} \frac{d}{d t} \tilde{\chi}_{l}^{(1)}(t) d t\right] \\
& =-\operatorname{Im}\left[W_{l}^{*(1)} \frac{\chi_{l}^{(1)}(\Delta t)-\chi_{l}^{(1)}(0)}{i \Omega_{l}^{(1)}}\right] \text {, }
\end{aligned}
$$

since the integral term in the middle line of Eq. (A4) does not have an imaginary part, as it follows from Eq. (A3). [The relations for the auxiliary functions along the trajectories on the second PES is obtained from Eq. (A4) by replacing the corresponding indices.] To obtain Eq. (A1), we integrate Eq. (21) from $t=0$ to $t=\Delta t$ (when coefficients of differential equation are fixed to constant values), and use Eq. (A4).

Numerical approach is based on the time step integration of Eq. (A3) with the following approximation:

$$
2 \int_{0}^{\Delta t} e^{i \Omega_{l}^{(1)} t} \alpha(t) d t \simeq \frac{\left(e^{i \Omega_{l}^{(1)} \Delta t}-1\right)}{i \Omega_{l}^{(1)}}[\alpha(\Delta t)+\alpha(0)],
$$

which results in a midpoint estimation of the integral at $\Omega_{l}^{(1)}=0$, and properly accounts for the damping if the difference of the bath Hamiltonians is large. Then, using the inverse relation

$$
\chi_{l}^{(1)}(\Delta t)=e^{-i \Omega_{l}^{(1)} \Delta t} \widetilde{\chi}_{l}^{(1)}(\Delta t),
$$

we derive

$$
\begin{aligned}
\chi_{l}^{(1)}(\Delta t) \simeq & e^{-i \Omega_{l}^{(1)} \Delta t} \chi_{l}^{(1)}(0)+W_{l}^{(1)} \frac{\left(1-e^{-i \Omega_{l}^{(1)} \Delta t}\right)}{\Omega_{l}^{(1)}} \\
& \times\left[\alpha(\Delta t)+\alpha(0)+\tanh \left(\frac{\epsilon}{2 T}\right)\right]
\end{aligned}
$$

which is used to propagate the auxiliary functions along the trajectories on the first PES, while the corresponding equation for the auxiliary functions along the trajectories on the second PES is obtained from Eq. (A7) by the change of the indices, and is of the form

$$
\begin{aligned}
\chi_{m}^{(2)}(\Delta t) \simeq & e^{-i \Omega_{m}^{(2)} \Delta t} \chi_{m}^{(2)}(0)+W_{m}^{(2)} \frac{\left(1-e^{-i \Omega_{m}^{(2)} \Delta t}\right)}{\Omega_{m}^{(2)}} \\
& \times\left[\alpha(\Delta t)+\alpha(0)+\tanh \left(\frac{\epsilon}{2 T}\right)\right] .
\end{aligned}
$$

Then, substituting Eqs. (A7) and (A8) in Eq. (A1), we obtain

$$
\alpha(\Delta t) \simeq \frac{1}{1+A}\left[(1-A) \alpha(0)-A \tanh \left(\frac{\epsilon}{2 T}\right)-B\right],
$$

where the coefficients are

$$
\begin{aligned}
A= & \frac{1}{L} \sum_{l=1}^{L}\left[\frac{\left|W_{l}^{(1)}\right|}{\Omega_{l}^{(1)}}\right]^{2}\left[1-\cos \left(\Omega_{l}^{(1)} \Delta t\right)\right] \\
& +\frac{1}{M} \sum_{m=1}^{M}\left[\frac{\left|W_{m}^{(2)}\right|}{\Omega_{m}^{(2)}}\right]^{2}\left[1-\cos \left(\Omega_{m}^{(2)} \Delta t\right)\right],
\end{aligned}
$$

and

$$
\begin{aligned}
B= & \frac{1}{L} \sum_{l=1}^{L} \operatorname{Im}\left[W_{l}^{*(1)} \frac{\left(1-e^{-i \Omega_{l}^{(1)} \Delta t}\right)}{i \Omega_{l}^{(1)}} \chi_{l}^{(1)}(0)\right] \\
& +\frac{1}{M} \sum_{m=1}^{M} \operatorname{Im}\left[W_{m}^{*(2)} \frac{\left(1-e^{-i \Omega_{m}^{(2)} \Delta t}\right)}{i \Omega_{m}^{(2)}} \chi_{m}^{(2)}(0)\right] .
\end{aligned}
$$

The algorithm works as follows. First we start equilibrium simulations of many MD trajectories on both PESs, say 
$L$ trajectories on the first and $M$ trajectories on the second PESs, respectively. They can also be simulated in advance and stored for the subsequent use. At the first step the initial conditions $\alpha(0)$ and (24) are set up, where the nonadiabatic couplings are calculated from the starting points of the corresponding MD simulations [the statistical sums should be calculated before the propagation in time, in order to determine the value of $\epsilon$ in accordance with Eq. (26)]. Then we take the coordinates of the phase spaces for the next step and determine a set of the nonadiabatic couplings $W_{l}^{(1)}, W_{m}^{(2)}$ and the differences of the bath Hamiltonians $\Omega_{l}^{(1)}, \Omega_{m}^{(2)}$ along the corresponding MD trajectories. This allows to determine the coefficients $A$ and $B$, see Eqs. (A10) and (A11), and to find the value of $\alpha(\Delta t)$ through the use of Eq. (A9). The auxiliary functions are updated using Eqs. (A7) and (A8). The obtained values of $\alpha(\Delta t)$ and $\chi_{l}^{(1)}(\Delta t), \chi_{m}^{(2)}(\Delta t)$ then replace the corresponding values at $t=0$ to set up for the next time step.

The suggested numerical algorithm has low computation cost (apart from MD simulations) and works for multidimensional PESs without any modification. The number of the MD trajectories, necessary to sufficiently explore the phase spaces of the PESs, depends on the system under consideration, but, in general, should be of order of several hundreds or thousands. For the practical purpose it is also possible to perform several calculations for some large number of the MD trajectories, and improve the result summing up contributions (with the proper statistical weights, see Ref. 48 for more details).

\section{APPENDIX B: BROWNIAN DYNAMICS SIMULATIONS}

Our Brownian dynamics simulation is based on the following integration scheme:

$$
\begin{aligned}
\mathbf{r}(t+\Delta t)= & \mathbf{r}(t)+\Delta t c_{1} \mathbf{v}(t)+(\Delta t)^{2} c_{2} \mathbf{a}(t)+\delta \mathbf{r}^{G}, \\
\mathbf{v}(t+\Delta t)= & c_{0} \mathbf{v}(t)+\Delta t\left(c_{1}-c_{2}\right) \mathbf{a}(t)+\Delta t c_{2} \mathbf{a}(t+\Delta t) \\
& +\delta \mathbf{v}^{G}
\end{aligned}
$$

which reduces to the velocity Verlet algorithm in the limit of low frictions $\xi \rightarrow 0$. Here vectors $\mathbf{r}$, v, and a denote the position, velocity, and the acceleration of the particle, $\Delta t$ is the time step of simulation, while the coefficients $c_{0-2}$ are of the form

$$
c_{0}=e^{-\Delta t \xi}, \quad c_{1}=\frac{1-c_{0}}{\Delta t \xi}, \quad c_{2}=\frac{1-c_{1}}{\Delta t \xi},
$$

where $\xi$ is the friction. The random variables $\delta \mathbf{r}^{G}$ and $\delta \mathbf{v}^{G}$ are sampled from a bivariate Gaussian distribution (for each pair of vectorial components) with zero mean values, variances $\left(k_{B}=1\right)$

$$
\begin{aligned}
\sigma_{r}^{2} & =(\Delta t)^{2} \frac{T}{\mu} \frac{1}{\Delta t \xi}\left[2-\frac{1}{\Delta t \xi}\left(3-4 e^{-\Delta t \xi}+e^{-2 \Delta t} \xi\right)\right], \\
\sigma_{v}^{2} & =\frac{T}{\mu}\left(1-e^{-2 \Delta t} \xi\right),
\end{aligned}
$$

and the correlation coefficient

$$
c_{r v} \sigma_{r} \sigma_{v}=\frac{T}{\mu \xi}\left(1-e^{-\Delta t \xi}\right)^{2} .
$$

A freely available RANLIB package was used to generate pairs $\left(\gamma_{1}, \gamma_{2}\right)$ of the uniform random variates on the interval $(0,1)$. Then the pairs of independent random numbers from the normal distribution with zero mean and unit variance $\left(\zeta_{1}, \zeta_{2}\right)$ are obtained as ${ }^{70}$

$$
\zeta_{1}=\sqrt{-2 \ln \gamma_{1}} \cos 2 \pi \gamma_{2}, \quad \zeta_{2}=\sqrt{-2 \ln \gamma_{1}} \sin 2 \pi \gamma_{2} .
$$

Finally, the desired pairs $\left(\zeta_{1}^{\prime}, \zeta_{2}^{\prime}\right)$ of random variates, sampled from the bivariate Gaussian distribution with the parameters, given by Eqs. (B4)-(B6), are

$$
\zeta_{1}^{\prime}=\sigma_{r} \zeta_{1}, \quad \zeta_{2}^{\prime}=\sigma_{v}\left(c_{r v} \zeta_{1}+\sqrt{1-c_{r v}^{2}} \zeta_{2}\right)
$$

${ }^{1}$ M. Born and R. Oppenheimer, Ann. Phys. 84, 457 (1927).

${ }^{2}$ R. D. Levine and R. B. Bernstein, Molecular Reaction Dynamics and Chemical Reactivity (Oxford University Press, New York, 1987).

${ }^{3}$ J. M. Bowman and G. C. Schatz, Annu. Rev. Phys. Chem. 46, 169 (1995).

${ }^{4}$ D. G. Truhlar, B. C. Garrett, and S. J. Klippenstein, J. Phys. Chem. 100, 12771 (1996), and references therein.

${ }^{5}$ N. F. Mott and H. S. W. Massey, The Theory of Atomic Collisions (Oxford, Clarendon, 1949).

${ }^{6}$ M. S. Child, Molecular Collision Theory (Academic, London, 1974).

${ }^{7}$ L. Salem, Electrons in Chemical Reactions (Wiley Interscience, New York, 1982).

${ }^{8}$ M. Klessinger and J. Michl, Excited States and Photochemistry of Organic Molecules (VCH, New York, 1995).

${ }^{9}$ V. May and O. Kühn, Charge and Energy Transfer Dynamics in Molecular Systems (Wiley-VCH, Berlin, 2000).

${ }^{10}$ A. Bjerre and E. E. Nikitin, Chem. Phys. Lett. 1, 179 (1967).

${ }^{11}$ J. C. Tully and R. K. Preston, J. Chem. Phys. 55, 562 (1971).

${ }^{12}$ L. D. Landau, Phys. Z. Sowjetunion 1, 88 (1932); C. Zener, Proc. R. Soc. London, Ser. A 137, 696 (1932).

${ }^{13}$ E. C. G. Stückelberg, Helv. Phys. Acta 5, 369 (1932).

${ }^{14}$ N. Rosen and C. Zener, Phys. Rev. 40, 502 (1932).

${ }^{15}$ L. D. Landau and E. Teller, Phys. Z. Sowjetunion 10, 34 (1936).

${ }^{16}$ V. K. Bykhovskii, E. E. Nikitin, and M. Y. Ovchinnikova, Zh. Eksp. Teor. Fiz. 47, 750 (1964).

${ }^{17}$ Y. N. Demkov, Sov. Phys. JETP 18, 138 (1964).

${ }^{18}$ V. I. Osherov and A. I. Voronin, Phys. Rev. A 49, 265 (1994).

${ }^{19}$ H. Nakamura and C. Zhu, Comments At. Mol. Phys. 32, 249 (1996).

${ }^{20}$ E. E. Nikitin, Annu. Rev. Phys. Chem. 50, 1 (1999).

${ }^{21}$ A. D. McLachlan, Mol. Phys. 8, 39 (1964).

${ }^{22}$ H.-D. Meyer and W. H. Miller, J. Chem. Phys. 70, 3214 (1979).

${ }^{23}$ G. D. Billing, J. Chem. Phys. 99, 5849 (1993).

${ }^{24}$ K. Drukker, J. Comput. Phys. 153, 225 (1999), and references therein.

${ }^{25}$ J. C. Tully, J. Chem. Phys. 93, 1061 (1990).

${ }^{26}$ S. Hammes-Schiffer and J. C. Tully, J. Chem. Phys. 101, 4657 (1994).

${ }^{27}$ O. V. Prezhdo and P. J. Rossky, J. Chem. Phys. 107, 825 (1997).

${ }^{28}$ D. Kohen, F. H. Stillinger, and J. C. Tully, J. Chem. Phys. 109, 4713 (1998).

${ }^{29}$ U. Müller and G. Stock, J. Chem. Phys. 107, 6230 (1997).

${ }^{30}$ N. Makri, Annu. Rev. Phys. Chem. 50, 167 (1999), and references therein.

${ }^{31}$ W. H. Miller, J. Phys. Chem. A 105, 2942 (2001), and references therein.

${ }^{32}$ A. G. Redfield, IBM J. Res. Dev. 1, 19 (1957); Adv. Magn. Reson. 1, 1 (1965).

${ }^{33}$ K. Blum, Density Matrix Theory and Applications (Plenum, New York, 1981).

${ }^{34}$ I. V. Alexandrov, Z. Naturforsch. A 36A, 902 (1981).

${ }^{35}$ W. Boucher and J. Traschen, Phys. Rev. D 37, 3522 (1988).

${ }^{36}$ A. Anderson, Phys. Rev. Lett. 74, 621 (1995).

${ }^{37}$ R. Kapral and G. Cicotti, J. Chem. Phys. 110, 8919 (1999).

${ }^{38}$ J. M. Jean, R. A. Friesner, and G. R. Fleming, J. Chem. Phys. 96, 5827 (1992).

${ }^{39}$ A. K. Felts, W. T. Pollard, and R. A. Friesner, J. Phys. Chem. 99, 2929 (1995). 
${ }^{40}$ U. Kleinekathöfer, I. Kondov, and M. Schreiber, Chem. Phys. 268, 121 (2001).

${ }^{41}$ A. Kühl and W. Domcke, J. Chem. Phys. 116, 263 (2002).

${ }^{42}$ S. Hahn and G. Stock, J. Chem. Phys. 116, 1085 (2002).

${ }^{43}$ M. V. Basilevsky, A. V. Soudackov, and A. I. Voronin, Chem. Phys. 235, 281 (1998).

${ }^{44}$ J. Casado-Pascual, I. Goychuk, M. Morillo, and P. Hänggi, Chem. Phys. Lett. 360, 333 (2002).

${ }^{45}$ M. Santer, U. Manthe, and G. Stock, J. Chem. Phys. 114, 2001 (2001).

${ }^{46}$ A. Sergi and R. Kapral, J. Chem. Phys. 118, 8566 (2003).

${ }^{47}$ M.-L. Zhang, S. Zhang, and E. Pollak, J. Chem. Phys. 119, 11864 (2003).

${ }^{48}$ A. A. Neufeld, D. Schwarzer, J. Schroeder, and J. Troe, J. Chem. Phys. 119, 2502 (2003).

${ }^{49}$ P. A. Frantsuzov, Chem. Phys. Lett. 267, 427 (1997).

${ }^{50}$ P. A. Frantsuzov, J. Chem. Phys. 111, 2075 (1999).

${ }^{51}$ H. Eyring, J. Chem. Phys. 3, 107 (1935).

${ }^{52}$ M. G. Evans and M. Polanyi, Trans. Faraday Soc. 31, 875 (1935).

${ }^{53}$ J. C. Tully, J. Chem. Phys. 61, 61 (1974).

${ }^{54}$ G. E. Zahr, R. K. Preston, and W. H. Miller, J. Chem. Phys. 62, 1127 (1975).

${ }^{55}$ P. M. Felker and A. H. Zewail, J. Phys. Chem. 89, 5402 (1985).

${ }^{56}$ J. C. Lorquet and B. Leyh-Nihant, J. Phys. Chem. 92, 4778 (1988).
${ }^{57}$ A. A. Neufeld, J. Chem. Phys. 122, 164110 (2005), preceding paper. ${ }^{58}$ A. A. Neufeld, J. Chem. Phys. 119, 2488 (2003).

${ }^{59}$ A. B. Doktorov and J. B. Pedersen, Appl. Magn. Reson. 26, 41 (2004).

${ }^{60}$ A. A. Neufeld, J. Chem. Phys. 121, 2542 (2004).

${ }^{61}$ For instance, these features are provided by the CPMD package, OIBM Corp. 1990-2004, OMPI für Festkörperforschung Stuttgart 1997-2001, http://www.cpmd.org

${ }^{62}$ M. P. Allen and D. J. Tildesley, Computer Simulation of Liquids (Clarendon, Oxford, 1996).

${ }^{63}$ T. P. Straatsma and J. A. McCammon, Annu. Rev. Phys. Chem. 43, 407 (1992).

${ }^{64}$ W. F. van Gunsteren, X. Daura, and A. E. Mark, Helv. Chim. Acta 85, 3113 (2002)

${ }^{65}$ W. W. Parson, Z. T. Chu, and A. Warshel, Biophys. J. 74, 182 (1998).

${ }^{66}$ R. A. Marcus, J. Chem. Phys. 24, 966 (1956).

${ }^{67}$ V. G. Levich and R. R. Dogonadze, Dokl. Akad. Nauk SSSR 124, 123 (1959) [Sov. Phys. Dokl. 124, 9 (1959)] .

${ }^{68}$ J. Casado-Pascual, M. Morillo, I. Goychuk, and P. Hänggi, J. Chem. Phys. 118, 291 (2003).

${ }^{69}$ J. Tang, Chem. Phys. 188, 143 (1994).

${ }^{70}$ G. E. Box and M. E. Miller, Ann. Math. Stat. 29, 610 (1958). 